\title{
The Evolution of the Nervous System as Model for Search New Pharmacological Strategies in Human Neurological Condition
}

\author{
Luisetto Mauro $^{1 *}$, G. Ibrahim ${ }^{2}$ and Oleg Latyschev ${ }^{3}$ \\ ${ }^{1}$ IMA academy, branch NATURAL SCIENCE, applied pharmacologist, Italy \\ ${ }^{2}$ Alexandria University Faculty of Science Department of Zoology, Marine Biology, Egypt \\ ${ }^{3}$ PRESIDENT IMA ACADEMY, Italy
}

*Corresponding author: Luisetto Mauro, Branch NATURAL SCIENCE , applied pharmacologist, Italy

\section{ARTICLE INFO}

Received: 幽 October 01, 2019

Published: October 11, 2019

Citation: Luisetto Mauro, G. Ibrahim, Oleg latyschev. The Evolution of the Nervous System as Model for Search New Pharmacological Strategies in Human Neurological Condition. Biomed J Sci \& Tech Res 21(5)-2019. BJSTR. MS.ID.003672.

\section{ABSTRACT}

\section{Nervous System Functions}

The nervous system directs and coordinates our movements. It receives stimuli from the environment around us and from all our internal organs. It interprets these stimuli and elaborates responses, which are transmitted to muscles or glands. To compare the nervous systems of invertebrates to vertebrates and between the various vertebrates. Can be an useful instrument in field of neuroscience, molecular biology, forensic science, and pharmacology, regenerative medicine.

Keywords: Nervous Systems; Vertebrates; Invertebrates; Neuroscience; Evolutive; Research; Neurodegenerative; Forensic Sciences

\section{Introduction}

\section{The Evolution of the Nervous System ( by G. Ibrahim )}

In the lower multicellular animals, such as porifers or sponges, there is no rudiment of nerves. We begin to see neurons, cells that conduct nerve stimuli, in coelenterates. In cnidarian polyps these cells appear scattered throughout the body, forming a network without much organization. There is no nerve center in these animals that runs this network. Each external stimulus acting on a point on the body is accompanied by a merely local response, determining a nerve impulse that propagates with decreasing intensity as it moves away from the stimulus's starting point. Cnidaria have a diffuse nervous system. Cnidarian phylum (corals, anemones, hydras and jellyfish). The more primitive Porifers (sponges) do not have a nervous system. In Cnidaria, there is a disordered network of neurons. And if a nerve pulse is triggered in one of them, it is transmitted to all cells that communicate with it through synapses, and from these to others, resulting in poorly elaborated responses - such as "pulsating" movements in living water when it's swimming. It is the most primitive type of nervous system, called Diffuse Nervous System. In flatworm worms (such as planar worms, for example), neurons associate together to form nerve threads attached to some structures - the nerve ganglia in the head. These ganglia already represent precarious nerve centers in coordinating body activities. In each ganglion there is a higher concentration of neurons.

The ganglionic nervous system begins to perfect in the annelids. In them, there is a larger conglomeration of neurons in the head, forming the cerebroid ganglia, which play a primitive brain role in commanding the other ganglia. From the cerebroid ganglia arise the periesophageal ganglia, which relate to a double ventral ganglionic nerve chain. Along this chain there are a pair of ganglia for each body segment [1-3]. These ganglia also have marked autonomy over the specific activities of the surrounding body area. In annelids, notwithstanding the presence of cerebroid ganglia, the pairs of ganglia along the ventral nerve chain have a great deal of autonomy, so a worm, even after being cut in half, continues to move the two pieces apart. The cerebroid ganglia are even more developed in arthropods, especially insects. In bilateral symmetry invertebrates (Platelminths, Nematelmints, Annelids, Molluscs and 
Arthropods) the nervous system is located in the ventral region of the body and is organized as one or more longitudinal nerve cords presenting two or more nerve ganglia, whether functioning as command centers along its length. In the possessors of many nerve ganglia, those in the anterior region - cerebroid ganglia - are more developed and function as a rudimentary brain that controls the other ganglia. This type of nervous organization is called the ganglionic nervous system.

\section{In Mollusca}

The nervous system is centralized and ganglionic, with three parts of nerve ganglia from which nerves go to different parts of the body. Sensory, visual, tactile, chemoreceptor and balance structures are present. The cephalopods have a large cerebroid ganglion that resembles the brain of vertebrates.

\section{In gastropods}

The nervous system consists of a set of ganglia and cords that are distributed throughout the body and innervate the different organs. The set of sensory organs comprises eyes, tentacles, asphradium and statocysts. The eyes, in the most primitive forms, are located at the ends of the tentacles and consist of simple depressions containing pigment and photoreceptor cells. In more advanced gastropods, depression closes, and a cornea and a lens are distinguished. The tentacles have eyes and tactile and chemoreceptor cells. Statocysts are important sensory cells for balance [3,4]. Aavailable only in species with gills, appears to function as an olfactory and chemoreceptor organ. Take a close note: The ganglionic nervous system, which characterizes invertebrates, has its double chain of lymph nodes arranged ventrally in the animal, that is, running along the ventral surface of the body. This system is in stark contrast to the vertebrate brain-spinal nervous system that we will see next. The cerebrospinal nervous system is in the dorsal position, descending from the head along the back of the animal.

\section{The Cerebrospinal Nervous System}

In vertebrates (fish, amphibians, reptiles, birds and mammals) the nervous system is well developed and is classified as a cerebrospinal nervous system. It is made up of a "thirst" - the CNS (central nervous system) - and a network of nerves that break out and distribute throughout the body - the peripheral nervous system.

\section{The Central Nervous System}

Vertebrates: The central nervous system is formed by the brain and spinal cord. The brain, in turn, comprises the following portions: brain, cerebellum, protuberance (pons or menencephalon) and bulb. In the lower vertebrates, from fish to birds, the cerebral hemispheres have a smooth surface. Therefore, such animals are considered lissencephalon (smooth brain). In mammals, however, grooves and circumvolutions appear, giving the brain a surface full of undulations [5]. For this reason, mammals are called gyrencephalon (brain with turns or curves). This transformation brought a great advantage for mammals: At the same volume, a circumvoluted brain has a considerably larger surface than if it had smooth hemispheres. As it is on the surface of the brain (cerebral cortex, with gray matter) that lie the bodies of neurons, the more grooves and convolutions the brain has, the larger its cortex, the larger the number of neurons, and thus the more efficient and improved it is.

The gray matter is located on the surface of the brain and is where the bodies of neurons accumulate. It is in them that the information is stored, the senses are perceived, the data obtained from external stimuli are "processed". Also, from the neurons depart the orders for muscle contractions or for glandular secretions etc. This superficial area is the cerebral cortex. It has the greatest importance in the degree of development of a species. The cerebral cortex is all divided into zones, like a map. Each area (some small, some large) represents a nerve center. Nervous centers are numerous throughout the brain, such as the centers of sight, hearing, smell, taste, pain, hunger, cough, tickling, anger, motor coordination (this is very wide and subdivides into areas corresponding to the various points of the body), the visual association for reading, in addition to the centers of respiratory, cardiac regulation, the thermoregulatory center, etc. The cortex is, as it turns out, the "seat" of control of conscious and unconscious acts as well as intelligence. The brain of a crocodile is, of course, larger than a brain of a mouse. However, the crocodile, as a reptile, is lissencephalon, while the mouse, as a mammal, is a gyrencephalon [6]. Therefore, the extension of the cerebral cortex of the mouse is larger than that of the crocodile, justifying greater rodent intelligence. That is why, in circuses, animal shows predominantly exhibit mammals.

In the deepest region of the brain lies the white mass. In it, there are practically no bodies of neurons, but only their branches (dentites and axons). The cerebellum, pons, and bulb are also very important because they enclose nerve centers that regulate various functions of relevant role. Breath and temperature controls are in the bulb. Control of body balance is in the cerebellum. Aside from the brain, the remainder of the CNS consists of the spinal cord (or spinal cord). It is a long cord of nervous structure that runs along the dorsum inside the spinal canal. It is therefore protected to its full extent by the spine. In it, the gray mass (as opposed to the brain) is in the center and the white mass in the periphery. The brain and spinal cord are fully protected by bone structures (the skull and spine) and by three outer membranes or meninges:

a) Dura mater - outermost, thick and fibrous

b) Arachnoid - has a vascularization that resembles a spider web

c) Pia mater - the most internal, thin and adherent to the CNS.

Below the arachnoid is the cerebrospinal fluid or cerebrospinal fluid, which has a protective function, involving the entire CNS. The spinal cord does not fully occupy the vertebral canal. It ends at about 
the level of the 1st or 2 nd lumbar vertebra. Spinal anesthesia, which worries so many people, offers no danger of traumatizing the spinal cord because it is made with a needle that penetrates the spinal canal below the 2 nd lumbar vertebra. Below this level is only one very thin fibrous cord - the filum terminale - that attaches the lower end of the medulla to the coccyx. Thus, the marrow should always be stretched. On its way, the spinal cord emits the spinal nerves, always in pairs. And you may notice that these nerves are closely related to gray matter. Many reflex acts are controlled directly by the spinal cord without brain interference. But in most cases, nerve stimuli reaching this organ are then transmitted to the brain, first reaching the diencephalon (region covering the hypothalamus) and then radiating to the most varied areas of the brain.

\section{The Peripheral Nervous System}

The peripheral nervous system consists of the internal network of nerves that depart from the CNS and are distributed throughout the body (motor nerves) and from nerves that come from all areas of the body and converge on the CNS (sensory nerves). Of course, there are mixed nerves whose characteristics include those of all types mentioned above, that is, they carry all orders of the CNS to the various points of the body and at the same time transmit the sensory perceptions of those same points to the CNS. We can then say that the PNS (peripheral nervous system) comprises all the nerves in our body. Many of these nerves act on the will of the individual, revealing voluntary action. These voluntary action motor nerves, along with the sensory nerves (which allow us to see, hear, feel pain, smell, taste, heat or cold etc.), offer the individual the possibility to relate to the environment. Therefore, they form what we may call the nervous system of relationship life. This system contrasts with another large number of nerves that act without the individual's conscience or will, regulating the activity of numerous organs such as the heart, stomach, intestines, diaphragm movements, salivary gland secretions, the pupil diameter etc. These involuntarily acting nerves, which work without one even suspecting, together form the autonomic nervous system or the vegetative life nervous system.

There are lesions that destroy areas of the CNS, completely nullifying the nervous system acting on the relationship life nervous system but leaving the nervous system of the vegetative life intact. When this occurs, the person becomes unrelated to the world around him and goes on to live an extremely vegetative life (the organs work well, but the individual seems to feel nothing or respond to external stimuli). It is common to call the nervous system of life a somatic nervous system relation (from the Greek soma, "body"), which does not seem very logical to us, since the autonomic nervous system, acting on the various parts of the body, is, consequently also somatic. The nervous system of relationship life comprises nerves that originate directly in the brain (particularly, the brain, cerebellum, pons or bulge, and, more numerously, the bulb) and nerves that originate in the spinal cord. We then distinguished cranial and spinal nerves, respectively. Cranial nerves are those that are born directly from the brain. In mammals they number 12 pairs (in other vertebrates there are only 10 pairs). Some are sensitive; others, engines; still others are mixed. All are cataloged by numbers. Often, a particular pair is referred to by its number, not by its name. Thus, it is mandatory to know the 12 pairs of cranial nerves by their order numbers:

1) Olfactory (sensitive): It transmits to the brain the impulses that give the perception of smell.

2) Optical (sensitive): Brings to the brain the impulses that provide the visual sensations.

3) Common Eye Engine or Oculomotor (engine): Moves the eyes up, down and in (nose direction).

4) Pathetic or Trochlear (motor): Makes the eyes rotate circularly.

5) Trigeminal (mixed): Perceives sensations of the face and acts on the muscles of the mimic.

6) Outboard Eye Engine or Abdulent (motor): Moves the eyes outward. Facial (mixed): It transmits the skin sensations of the face and also acts in mimicry.

7) Acoustic or Atrio-cochlear (sensitive): One of its branches leads to the brain impulses that will give sound perceptions. The other leads to the cerebellum impulses responsible for the notion of body balance.

8) Glossopharyngeal (mixed): Transmits the impulses that give the perception of taste and moves the tongue.

9) Pneumogastric or Vagus (mixed): It acts on the thoracic and abdominal organs and is the main nerve of the parasympathetic system.

10) Spinal, Spinal or Accessory (motor): Acts on the shoulder muscles (shoulder slapping of the naughty).

Hypoglossus (motor): Helps glossopharyngeal in tongue movement. They all act on organs and muscles from head to shoulder. Only the pneumogastric or vagus goes into the body and innervates the viscera, such as the heart, stomach, intestines and other organs. In fact, this is the only cranial pair that has involuntary action, therefore belonging to the autonomic nervous system. The spinal nerves are all born from the spinal cord, but they go to different parts of the body, such as arms, trunks and legs. They comprise 31 pairs and are all mixed, that is, they transmit sensations of the skin and organs to the spinal cord, as they transmit its motor orders to the muscles. Each spinal nerve contains sensory fibers, which bring to the medulla sensory perceptions of a region of the body, and motor fibers, which carry motor stimuli from the medulla to these regions. The spinal nerves emerge from the medulla through two roots - anterior root and posterior root - which join just below to form the nerve itself. Posterior roots (with sensory fibers) are afferent to the medulla, as they conduct the stimulus towards it. The anterior roots (with motor fibers) are efferent in relation to the 
medulla, because they carry stimuli that move away from it. To its greatest extent, therefore, each spinal nerve encloses sensory and motor fibers and proceeds as a "two-way road". From the sensory fibers come the perceptual stimuli and from the motor fibers the command commands.

Transition (or association) neurons can make the connection between a sensory neuron and a motor neuron: on the same side and at the same level as the gray matter of the medulla; opposite but at the same level in the medulla (horizontal cross-association); on the opposite side and at another level in the medulla (vertical cross association); on the same side and at another level (uncrossed vertical association). Surely you have ever touched a finger harder than you expected on the tip of a needle. And he withdrew his finger abruptly, so quickly that it would not be possible or consciously for him. This fact is an example of arc reflex. The reflex arc is the immediate response to arousal of a nerve without the interference of the individual's will (and sometimes even consciousness). In the above example, the stimulus ran through the sensory fibers of a spinal nerve, bypassed the gray matter of the spinal cord by the association neuron, and returned through the motor fibers of the spinal nerve, reaching the muscles of the arm and hand, causing them to contract. and remove the finger from the tip of the needle. Many reflexes are by medullary mechanism only.

The rotulian or patellar reflex, which the doctor investigates a small blow to the rotulian tendon (knee), denotes through the sudden response of the musculature, involuntarily kicking the air, that the spinal nerves of this region, as well as the medulla, are perfect and in good working order. But some reflexes are more complex and involve stimuli that go to and return to the cerebral cortex bringing orders to the marrow. At the level of the bulb, stimuli from one side of the body transfer to the opposite side of the brain, just as motors coming from one cerebral hemisphere cross at the level of the bulb or medulla. Across the body In a reflex involving the medulla and the brain, the sensory stimulus moves back and forth at the level of the bulb, but the motor response coming from the brain only reverts to the primitive side at the level of the medulla.

\section{The Autonomic Nervous System}

The autonomic nervous system or neurovegetative system is formed by nerves that work without any dependence on the will or conscience of the individual. These nerves are divided into two large groups: the sympathetic system and the parasympathetic system. Both groups are antagonistic. In the organ in which the sympathetic nerves act by stimulating, the parasympathetic nerves that will have there act inhibiting. In other organs, the parasympathetic is exciting and the sympathetic is inhibiting. The heart is stimulated by the sympathetic hair and inhibited by the parasympathetic. The opposite occurs, however, in the gut. From the antagonism of both groups, the functional balance of the organism arises. When one of the groups becomes disturbed, there is imbalance, and the organic functions begin to disturb. This explains neurovegetative disorders such as tachycardias (palpitations), vomiting, diarrhea, changes in blood pressure, salivation, etc. The nerves of the sympathetic system are all born from anterior branches of spinal nerves. The nerves of the parasympathetic system form, some also from anterior branches of spinal nerves, while others are born directly from the brain (these are the various branches of the pneumogastric or vagus).

The nerves that make up the ANS (autonomic nervous system) end in glands or smooth muscle tissue. They command, without the individual's conscience, the secretory activity and movements of the viscera in whose structure there are smooth muscles. Sympathetic parasympathetic Pupils dilate contracts Heart rate accelerates (tachycardia) Retards (bradycardia) Blood vessels contract (person becomes pale) Dilates (person turns red) Stomach paralyzes excites Intestine paralyzes excites Bladder relaxes contracts. The nerves of the anastomoses sympathetic system gather their roots and form the sympathetic plexuses, such as the cardiac plexus (which acts on the heart), the solar plexus or celiac plexus (whose branches will form in the liver, stomach and intestines). sacral plexus (which acts on the urogenital organs). All of these organs also receive nerve branches from the parasympathetic system.

\section{Materials and Methods}

After this introduction whit a bibliographic research some relevant literature reported in references is analysed in order to produce a global conclusion.

\section{Result}

Comparing the information reported in the introduction section whit some relevant literature related some human neurodegenerative condition is clear that compared anatomy and physiology is an useful instrument for base research and to produce new pharmacological strategy.

\section{Conclusion}

Is really crucial in today research, related the actual LOW efficacy in some neurodegenerative disease, to deeply use compared anatomy and physiology as source of real interesting information .The evolutive pattern as a key role in applied research. Zoology, biology, anatomy, physiology ,molecular biology , pharmacology as basic science for new Methodology in neurodegenerative disease research.

\section{References}

1) Luisetto M, Farhan Ahmad Khan, Ahmed Yesvi Rafa, Behzad Nili Ahmadabadi, Ghulam Rasool Mashori (2019) Decussatio pyramid and optical chiasm as an interesting example of evolutionary process useful in understanding some cervical spinal cord Phenomena 3(3).

2) Luisetto M, Naseer Almukhtar, Ghulam Rasool Mashori, Ahmed Yesvi Rafa, Farhan Ahmad Khan (2018) Journal of Neuroscience and Neurological Disorders Endogenous archeological sciences: Physiology, 
Neuroscience, Biochemistry, Immunology, Pharmacology, Oncology and Genetics as instrument for a new field of investigation? 2: 065-097.

3) Luisetto M, Ahmadabadi BN, Rafa AY, Sahu RK, Cabianca L, et al. (2019) The turing machine theory for some spinal cord and brain condition, A toxicological - antidotic depurative approach. J Neurosci Neurol Disord 3: $102-134$.

4) M Luisetto, Farhan Ahmad Khan, Luca Cabianca, Mobin Ibne Mokbul (2016) Amygdala pharmacology and crime behavior, dysfunctions to be considered as a disease? 2016 IABCR International Archives of

ISSN: 2574-1241

DOI: 10.26717/BJSTR.2019.21.003672

Luisetto Mauro. Biomed J Sci \& Tech Res

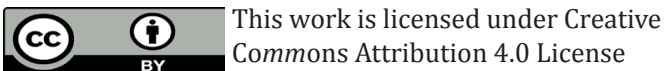

Submission Link: https://biomedres.us/submit-manuscript.php
BioMedical and Clinical Research 2(2).

5) M Luisetto, Farhan Ahmad Khan, Naseer Almukhtar (2019) Mindset Kinetics and Crime Behavior- Quantitative Methods? A New Forensic Quantitative Approach. How Biochemistry, Toxicology, Imaging Principle can Help in Jurisdictional Settings. Am J Biomed Sci Res 16(2).

6) M Luisetto, Naseer Almukhtar, Gamal Abdul Hamid, et al. (2019) Molecular Characterization of Brain and other Structures in Vertebrates and Invertebrates. Arch Neurol Neurosci 3(5).

$\begin{array}{ll}\text { BIOMEDICAL } & \text { Assets of Publishing with us } \\ \text { RESEARCHES } & \text { - Global archiving of articles } \\ & \text { - Immediate, unrestricted online access } \\ & \text { - Rigorous Peer Review Process } \\ \end{array}$

